\title{
Challenges in Implementing Surveillance Tools of High-Income Countries (HICs) in Low Middle Income Countries (LMICs)
}

\section{Kushlani Jayatilleke, MBBS, MD}

\section{Address}

Sri Jayewardenepura General Hospital, Nugegoda, Sri Lanka

Email: kjayatilleke@gmail.com

Published online: 28 August 2020

(C) Springer Science+Business Media, LLC, part of Springer Nature 2020

This article is part of the Topical Collection on Infection Prevention and Safety in Low and Middle Income Countries

Keyword Surveillance of communicable diseases - Surveillance in LMIC · HAI · AMR · Surveillance tools of HIC

\section{Abstract}

Purpose of Review Surveillance of communicable diseases is essential in all countries to prevent and control infections, to detect outbreaks and also to see the effects of interventions. The data should be reliable, and collection, analysis and feedback as well as the action based on this data should be fast. In this article, author discusses the limitations the Low Middle income Countries (LMICs) have in implementing disease surveillance and some suggestions for improvement.

Recent Findings Integrated Disease Surveillance and Response (IDSR) has been implemented successfully through most of the countries in Africa though they belong to low or LMIC. Major barriers for surveillance of Healthcare Associated Infections in LMICs are nonavailability of adequate number of healthcare personnel such as infection control personnel as well as not having an integrated healthcare system with an effective data flow. For some infections, not having proper diagnostic facilities is a major obstacle. An important capacity limitation in clinical laboratories of LMICs is identification of antimicrobial resistant organisms as well as other pathogens to species level. This affects the surveillance of infections and antimicrobial resistance.

Summary Use of modern technology, capacity building including the human resources as well as the laboratory capacity in healthcare setting, improving data communication methods, are the main recommendations made. Education and training of healthcare staff as well as educating the general public to change the attitudes of people is another aspect that we need to concentrate. 


\section{Background}

Surveillance of infections and antimicrobial resistance in the community as well as in hospitals is recommended to prevent and control infections and to reduce antibiotic resistance $[1,2]$. The surveillance data will be useful to guide infection prevention and control measures, to detect outbreaks and also to see the results of interventions [1].

Communicable disease surveillance in the community is essential for effective control of such diseases [3]. Healthcare Associated Infections (HAI) has high disease burden with associated mortality worldwide, but the impact is more in Low Middle income Countries (LMICs) [4]. Therefore, surveillance of communicable diseases in community as well as the healthcare associated infections in healthcare settings is extremely important in all countries.

LMICs have many limitations in relation to availability of resources for surveillance. This article is a review of the challenges faced by the LMICs in implementing surveillance tools recommended in high income countries (HICs) and opportunities to overcome these challenges.

\section{Surveillance Requirements}

\section{Legislation for Surveillance [5]}

It is important to have legislations and regulations in place to get surveillance done effectively.

\section{Surveillance Strategy [5]}

Different diseases may need different surveillance strategies based on the objective of the surveillance system. If the data is needed for early warning of an outbreak, fast reporting is needed than if it was needed for monitoring of a programme.

\section{Laboratory Capacity [6]}

This is crucial for communicable disease surveillance in most diseases and for surveillance of antimicrobial resistance.

\section{Implementers and Stakeholders [5]}

It is important to define the responsibilities of each person in the surveillance system. Trained staff in adequate numbers are essential for this purpose.

\section{Networking and Partnership [5]}

Communication between different sectors within the country as well as internationally is crucial. Tools for data communication such as information technology should be available for all participants.

\section{Surveillance methodologies: International}

Certain diseases, such as yellow fever, plague, and cholera as well as diseases which are unusual or unexpected and may have serious public health impact, are currently subject to the International Health Regulations [7]. The regulations, which were first adopted by the World Health Assembly in 1951 and then revised slightly in 1969, are a mechanism to provide security against the international spread of epidemic diseases with a minimum interference with world traffic. 
These are the only binding international legislation for public health, and they require reporting of certain diseases to World health Organization (WHO) within $24 \mathrm{~h}$ by the national health administration. These diseases should be reported by the health professionals to the next administrative level by a rapid method such as phone, e-mail, fax or telex [8].

In Centers for Disease Control and Prevention (CDC), USA, The Public Health Data Modernization Initiative was started to improve data by modernizing tools, technology, strategy, and the attitudes of people and culture around data [9]. CDC uses cloud technology to monitor 4 million health messages from visits to emergency rooms every day to quickly detect events that may require a response. This initiative describes how the technology can be used to predict epidemics, understand, share and communicate data rapidly and effectively and to identify areas of common investments for the future [9].

In the USA, National Healthcare Safety Network (NHSN) is the most widely used HAI tracking system. It also monitors antimicrobial use and resistance, blood safety errors and important healthcare process measures such as healthcare personnel influenza vaccine status and infection control adherence rates [10].

European Centre for Disease Prevention and Control (ECDC) is conducting point prevalence survey of healthcare-associated infections (HAIs) and antimicrobial use (AU) in acute care hospitals in countries of European Union (EU) [11]. Data of HAI, Antimicrobial resistance (AMR) and important infectious diseases are published and accessible in ECDC website [12]. A weekly, electronic, peer-reviewed publication, Eurosurveillance provides timely facts and guidance for public health professionals and decision-makers in the field of infectious disease to facilitate the implementation of effective prevention and control measures [13].

\section{Implementational challenges in LMICs}

Most countries have communicable disease surveillance systems in place [1416]. Public health legislation and regulations, including IHR 2005 regulations governing notifiable diseases and other communicable diseases of public health importance, provide the regulatory framework for the implementation of surveillance and response systems. Some of these laws and regulations have become outdated and may require some amendments. Periodic review and evaluation will establish the relevance, adequacy and need for update [8].

Nepal is a low-income country which has a hospital-based sentinel surveillance system in 82 hospitals covering all 75 districts for reporting of selected water-borne, and food borne diseases in addition to a Health Management Information System (HIS). It focuses on weekly reporting of certain priority diseases. The traditional data collection methods are used where there is no HIS available $[17,18 \bullet \bullet]$.

National Surveillance Programme for Communicable Diseases (NSPCD) was launched in India in 1997-98 [19]. Integrated Disease Surveillance Project (IDSP) which was formally launched in 2004 with the aid of World Bank has improved since then; this too reports on priority communicable diseases. Though the infectious disease surveillance has improved since the introduction of IDSP, in state and district level, the monitoring and evaluation have been 
weak, and there is a shortage of human resources [20]. According to the National Centre for Disease Control, India, 133 outbreaks were reported through the IDSP from January 1 to February 4, 2018. Media scanning and verification cell daily receives an average of 2-3 media alerts of unusual health events which are detected and verified [21].

Though Sri Lanka is categorized as an upper-middle-income country, the surveillance is still carried out in traditional paper-based system, complemented by a web-based system for certain diseases such as dengue [22]. Though a webbased surveillance system was launched, most of the data is still sent through traditional paper-based method [23]. However, the integrated surveillance of communicable diseases is not happening in timely manner, and GPS and other novel technology are not available. Chandrashekar K et al., 2013, published an article which discusses the experience in the northern province of Sri Lanka and mentions that due to limiting source of notification and paper-based surveillance system, the data reporting is incomplete and slow. It also discusses how the laboratories can be linked to surveillance, to improve the completeness and timeliness of data reporting [24]. This paper highlights an important issue in countries such as Sri Lanka where different types of medical practitioners are present and without an integrated information system to cover all levels of healthcare systems. This becomes a major barrier for an effective surveillance system.

Integrated Disease Surveillance and Response (IDSR) has been implemented successfully through most countries in Africa, though they belong to low or LMICs [25••]. IDSR was implemented in 44 countries by December 2017, and $70 \%$ had electronic IDSR systems. However, the target of at least 90\% IDSR implementation coverage at the peripheral level was achieved only by 12 countries. Sixty-eight per cent of the countries have achieved timeliness and completeness threshold of at least $80 \%$ of reporting units. WHO supported this project. Modern technology such as GPS has not been used. This is a good example of where surveillance of communicable diseases is implemented effectively, in LMICs.

These examples indicate that implementation of communicable disease surveillance can be successful in LMICs if appropriate initiatives are taken. GPS and other modern technologies available can be used successfully in most LMICs [25••]. The problem often lies in attitudes of people, and not having a proper leadership along with monitoring plans. Whether people are ready to have such systems in place and how to get the support of the relevant authorities for such projects is a question for which a relevant answer is lacking. Adequate number of trained personnel should be made available carrying out such projects with proper facilities. Real-time monitoring, evaluation and feedback are essential for a successful surveillance programme.

Most LMICs have vector borne diseases, and therefore integration of epidemiological data with environmental and vector surveillance data will be very useful [26]. In Africa, mobile technology has been used successfully to carry out surveillance in humans and animals [27]. Current COVID 19 outbreak is another example which highlights the importance of strengthening the surveillance of communicable diseases in the community as well as in animals globally, to prevent disasters, large scale epidemics and pandemics. 
Another challenge in LMICs is the lack of properly linked electronic health records (EHRs) with data management and communication system between primary, secondary and tertiary care hospitals. This hinders the possibility of using the data from EHRs which appear to be a promising newer source of data for public health surveillance and for assessing the prevalence of disease or behavioural risk factors in the population seeking healthcare [28].

Surveillance of Healthcare Associated Infections (HAI) and Antimicrobial Resistance is recommended as a core component of Infection Prevention and Control [2].

In Sri Lanka, though there is surveillance of HAI at different levels in different hospitals, there is no central monitoring of the data. Certain parameters such as Staphylococcus aureus bacteraemia and MRSA bacteraemia rate, infection rate of lower segment Caesarean section (LSCS) and hand hygiene compliance rate have been identified as national indicators related to infection prevention and control activities in hospitals. However, these have not helped to improve the practices for many reasons [29].

Staphylococcus aureus bacteraemia and MRSA bacteraemia rate are calculated as per 10,000 patient days and does not reflect true HAIs as Staphylococcus aureus bacteraemia is not always associated with healthcare. This tends to give a wrong impression of healthcare quality and safety. In England, the Staphylococcus aureus bacteraemia rate per 100,000 population is calculated and helps in determining the prevalence of $S$ aureus in the population [30]. Adopting the same parameter in countries such as Sri Lanka, where there is no proper referral mechanism in the health system and thus no possibility of tracking of actual footfalls to each hospital, does not work. The feedback and action based on this data is not helpful in containing the infection optimally. However, the Director Quality and Safety of the Ministry of Health collects this data through the quality units of the hospitals. This data cannot be used for benchmarking purposes and to evaluate the effectiveness of Infection Prevention and Control practices because the differences in types of hospitals and case mix and differences in units are not considered. Therefore, not much effective action can be taken based on this data. These problems are also common to the Staphylococcus aureus bacteraemia surveillance in the UK [30]. If the clinical data of these patients are also collected and evaluated, for example, what proportion are due to central line associated infections etc., some useful intervention could have been taken based on this data. Surveillance of surgical site infections is not very successful in LMICs where the health systems are not well organized and the patients can be admitted in different tiers of healthcare institutions with infections which may not have proper reporting mechanisms.

Similarly very little information is available on HAI data from Africa [31]. In South Africa, inadequate human resources and manual data entry were two main reasons given for poor HAI surveillance [32].

Absence of a national programme for monitoring infection prevention and control activities in healthcare settings manned with dedicated experts is one of the major problems faced by most LMICs. 
Surveillance of HAIs such as Central-line Associated Bloodstream Infections (CLABSI), Ventilator Associated pneumonia (VAP), Catheter Associated Urinary Tract Infections (CAUTI) and Antibiotic Associated Diarrhoea caused by Clostridium difficile are negligible in most LMICs [33]. One reason for this is the inadequacy of infrastructure and human resources such as trained staff to collect and report appropriate data. An example for this is calculation of urinary catheter days for CAUTI in non-intensive care setting. Manual collection becomes difficult as this is not automated and allocated healthcare staff are generally overloaded with routine care. Most hospitals do not fulfil the minimum requirement of 1:250 ratio between the trained dedicated full-time infection preventionist (IP) and the number of hospital beds [2]. Computerized data management systems which have the capability to capture these data are generally not available.

If the diagnostic algorithms of HAI of HIC are considered, most of them are not practical in LMIC. One such example is diagnosis of infection due to Clostridium difficile $[34 \bullet \bullet, 35 \bullet \bullet$. Most hospitals in LMICs do not have facilities to detect Clostridium difficile by nucleic acid amplification test [NAAT] or toxins $\mathrm{A}$ and $\mathrm{B}$, thus the diagnosis may be missed. Limitations in laboratory capacity are major constrains in diagnosing and reporting infections.

With the rising problem of antimicrobial resistance, it is essential to do surveillance on antimicrobial-resistant pathogens. To strengthen knowledge through surveillance and research is one of the 5 strategic objectives identified by the WHO in its global action plan on antimicrobial resistance [36]. Good quality clinical microbiology laboratory facilities are essential for this purpose.

Clinical microbiology laboratory services with blood culture facilities are available only at national and provincial level hospitals and the peripheral healthcare facilities (PHF) are poorly linked to these facilities in majority LMICs $[37 \bullet \bullet]$. Most patients are started on antibiotics without collecting the specimens for culture in PHF due to the unavailability and unaffordability of microbiological investigations such as cultures. This leads to missing the opportunity for making an aetiological diagnosis as well as identifying antimicrobial resistance. The optimum number of specimens for blood cultures may not be collected due to high cost, leading to reduction in chance of isolating the pathogen as well as missing opportunities for diagnosing infections such as endocarditis. Most clinical microbiology laboratories of LMICs do not have automated blood culture systems which again lead to reporting delays resulting in inappropriate empirical use of broad-spectrum antibiotics [38].

Another important capacity limitation in clinical laboratories is identification of antimicrobial-resistant organisms as well as other pathogens to species level. This adversely affects the surveillance of infections and antimicrobial resistance. Only few laboratories have capacity to identify pathogens to species level by using automated systems such as a Vitek 2 or BD Phoenix technology. Even if it is available, they are usually used for blood culture and sterile fluid isolates only. Use of this technology for all isolates such as from urine culture becomes expensive and unaffordable. Nowadays matrix-assisted laser desorption/ionization-time-of-flight (MALDI-TOF) is a method available in most clinical laboratories in developed countries to identify micro-organisms with much less cost per test [39]. As the initial investment is very high, use of this technology is limited in most clinical laboratories of LMICs. 
Detection of Minimum Inhibitory Concentration (MIC) to different antibiotics is essential for identifying AMR [40]. According to CDC, Staphylococcus aureus strains for which the vancomycin MIC is $\geq 4 \mu \mathrm{g} / \mathrm{ml}$ should not be discarded until the MICs have been confirmed by a validated method [41]. In majority of the laboratories, facilities to perform MICs are not available, therefore missing the resistant pattern. Adequate facilities for storing these important isolates are also inadequate in smaller laboratories thereby evaluation of such isolates by reference laboratories is also often missed. This is also true for some tertiary care hospital laboratories as capacity is limited and appropriate guidelines are not available along with facilities for storage.

Facilities to perform molecular techniques such as pulsed-field gel electrophoresis and whole genome sequencing are not available in reference laboratories in countries such as Sri Lanka. Most laboratories are not accredited by a third party due to the high cost, though Sri Lanka Accreditation Board has accredited limited number of clinical laboratories in Sri Lanka mainly in the private sector [42].

Data collection, analysis and reporting of AMR are inconsistent in countries such as Sri Lanka [42]. One reason for this is that most laboratories do not have well planned laboratory information management systems (LIMS) which are linked to data analysis platforms such as WHONET (https://www.who.int/ medicines/areas/rational_use/AMR_WHONET_SOFTWARE/en/). Commercial LIMS are unaffordable in countries such as Sri Lanka, and most laboratories use either manual paper-based data bases or locally developed LIMS which are not validated as suitable for data management. WHONET is a Windows-based database software approved by the WHO which is for management and analysis of microbiology laboratory data with a special focus on the analysis of antimicrobial susceptibility test results. This can be downloaded and used free of charge. Currently this is used in over 90 countries in clinical as well as other laboratories. Baclink can transfer data into WHONET from common commercial database and spreadsheet software, commercial susceptibility test instruments for MIC broth microdilution and disk diffusion readers and hospital and laboratory information systems through text files (https://www.who.int/ medicines/areas/rational_use/AMR_WHONET_SOFTWARE/en/). Automatic alerts and daily summary reports of analysed AMR data can be generated through this (https://www.who.int/medicines/areas/rational_use/AMR_ WHONET_SOFTWARE/en/). This also generates reports to be submitted to Global Antimicrobial Resistance Surveillance System (GLASS) of WHO. Though WHONET is used by some laboratories in LMICs, it is not used optimally.

In 2018, globally 55\% of pulmonary TB cases were bacteriologically confirmed. In HICs with widespread access to the most sensitive diagnostic tests, about $80 \%$ of pulmonary TB cases are bacteriologically confirmed. As some cases of rifampicin resistance may be missed in TB if only Xpert MTB/RIF is used, it is recommended to introduce whole genome sequencing into surveillance activities, particularly in south-eastern Africa [43]. Despite increases in TB notifications, there is still a large gap between the estimated number of incident cases (9.0-11.1 million globally in 2018) and the number of new cases reported (7.0 million), due to a combination of underreporting of detected cases and underdiagnosis (if people with TB do not access healthcare or are not diagnosed when they do). Ten countries accounted for about $80 \%$ of the gap, 


\section{Conclusions}

with India (25\%), Nigeria (12\%), Indonesia (10\%) and the Philippines (8\%) accounting for more than half of the total. In these countries in particular, intensified efforts are required to reduce underreporting of detected TB cases and improve access to diagnosis and treatment [43].

- Most of the surveillance tools implemented in surveillance of communicable diseases in the community in HICs can be used in LMICs as in Africa $[25 \bullet \bullet]$.

- Major setback for implementing HAI surveillance in LMICs is not having adequate number of trained staff and a national programme for infection prevention and control in healthcare setting, who should carry out the national surveillance and feedback. Manual method of data collection is not suitable with the high workload of the staff which makes data collection, especially the denominator data for calculating HAI rates, impractical. Limited laboratory facilities also make the HAI surveillance incomplete in these countries.

- Limited laboratory capacity is a major challenge faced in antimicrobial resistance surveillance in LMICs. Not having facilities for identification of pathogens to species level, for performing reliable methods of minimum inhibitory concentrations and not having accredited laboratories, is a common problem in laboratories of LMICs.

- Not having well planned LIMS which will export AMR data to a software such as WHONET which can be used to create summary reports and alerts of importance routinely is another limitation in LMICs.

- Integrated Surveillance Systems should be implemented in all countries using modern technology to improve the speed of detection and control of outbreaks and to implement infection prevention and control measures in community setting.

- Capacity building in healthcare with adequate number of trained healthcare workers and infection preventionists will be important to improve the surveillance of healthcare associated infections.

- Improving laboratory capacity with proper quality assurance programmes is important to support communicable disease surveillance in the community as well as for surveillance of HAI and AMR.

- Data communication methods among all types and levels of healthcare institutions and the national and regional surveillance centres should be improved in LMICs. 
- National Programme should be in place for Infection Prevention and Control which should have dedicated specialists in IPC to implement HAI surveillance.

- Well planned LIMS should be available in LMICs where routine antimicrobial data analysis with automatic generation of summary reports and alerts is implemented through WHONET or other similar software.

\section{Compliance with Ethical Standards}

Conflict of Interest

Kushlani Jayatilleke declares that she has no conflict of interest.

Human and Animal Rights and Informed Consent

This article does not contain any studies with human or animal subjects performed by any of the authors.

\section{References and Recommended Reading}

Papers of particular interest, published recently, have been highlighted as:

- Of importance

$\bullet \quad$ Of major importance

1. WHO Recommended Surveillance Standards. Second edition; produced jointly by technical programmes in WHO and by UNAIDS; World Health Organization; Department of Communicable Disease Surveillance and Response; https://www.who.int/csr/resources/ publications/surveillance/whocdscsrisr992.pdf.

2. Guidelines on core components of infection prevention and control programmes at the national and acute health care facility level; WHO; ISBN: ISBN 978-92-4154992-9; November 2016; https://www.who.int/ gpsc/ipc-components/en/.

3. Public Health Systems and Emerging Infections: Assessing the Capabilities of the Public and Private Sectors: Workshop Summary; Institute of Medicine (US) Forum on Emerging Infections; Editors: Jonathan $\mathrm{R}$ Davis and Joshua Lederberg. Washington (DC): National Academies Press (US); 2000. ISBN-10: 0-30906829-0; https://www.ncbi.nlm.nih.gov/books/ NBK100253/.

4. Allegranzi B, Nejad SB, Combescure C, Graafmans W, Attar H, Donaldson L, et al. Burden of endemic healthcare-associated infection in developing countries: systematic review and meta-analysis. Lancet. 377(9761):228-41, January 15, 2011; Published: December 10, 2010. https://doi.org/10.1016/S01406736(10)61458-4.

5. Guide to monitoring and evaluating communicable disease surveillance and response systems; WHO/CDS/ EPR/LYO/2006.2; World Health Organization 2006;

6. Epidemiology and Laboratory Capacity for Prevention surveillance/WHO_CDS_EPR_LYO_2006_2.pdf. and Control of Emerging Infectious Diseases (ELC); https://www.cdc.gov/ncezid/dpei/epidemiologylaboratory-capacity.html.

7. International health regulations (2005) - 2nd ed.; 1 . Legislation, Health. 2. Communicable disease control legislation. 3. Disease notification - legislation. 4. International cooperation. I. World Health Organization. II.Title: IHR (2005). ISBN 978924158041 0; https:// apps.who.int/iris/bitstream/handle/10665/43883/ 9789241580410_eng.pdf;jsessionid= FE6748233C837F5C61D023ED8944B4F1?sequence=1.

8. WHO Report on Global Surveillance of Epidemicprone Infectious Diseases - Introduction; https://www. who.int/csr/resources/publications/introduction/en/ index4.html.

9. Public Health Surveillance and Data; Centers for Disease Control and Prevention, Deputy Director for Public Health Science and Surveillance (DDPHSS); https://www.cdc.gov/surveillance/surveillance-datastrategies/data-IT-transformation.html

10. HAI data; Centers for Disease Control and Prevention (CDC); https://www.cdc.gov/hai/data/index.html.

11. European Centre for Disease Prevention and Control. Point prevalence survey of healthcare associated infections and antimicrobial use in European acute care hospitals - protocol version 5.3. Stockholm: ECDC; 
2016; https://www.ecdc.europa.eu/sites/portal/files/ media/en/publications/Publications/PPS-HAIantimicrobial-use-EU-acute-care-hospitals-V5-3.pdf.

12. European Centre for Disease Prevention and Control. Tools; https://www.ecdc.europa.eu/en/data-tools.

13. Eurosurveillance Europe's journal on infectious disease surveillance, epidemiology, prevention and control; https://www.eurosurveillance.org/.

14. Comprehensive Assessment of National Surveillance Systems in Sri Lanka; Joint Assessment Report 4-13 March 2003, World Health Organization Regional Office for South-East Asia New Delhi February 2004.

15. Integrated Disease Surveillance Programme, Ministry of Health \& family welfare, Government of India; https://idsp.nic.in/.

16. Update: Integrated Diseases Surveillance and Response implementation in Ethiopia. https://www.who.int/ countries/eth/areas/surveillance/en/idsr_ implementation.pdf.

17. Government of Nepal, Ministry of Health and Population, Department of Health Services, Epidemiology and Disease Control Division Kathmandu, Nepal http://www.edcd.gov.np/section/surveillance-ofcommunicable-disease-program.

18.• A guide to Early warning and reporting system (EWARS); Government of Nepal, Ministry of Health and Population, Department of Health Services, Epidemiology and Disease Control Division, Teku, Kathmandu, Nepal; http://www.edcd.gov.np/resources/ download/a-guide-to-ewars-2019.

Well planned early warning and reporting system from a LMIC.

19. World Bank Country and Lending Groups; Country Classification; https://datahelpdesk.worldbank.org/ knowledgebase/articles/906519-world-bank-countryand-lending-groups.

20. Raut DK, Bhola AK. Integrated Disease Surveillance in India: Way Forward. Glob J Med Publ Health (GJMEDPH). 2014;3(4).

21. Integrated Disease Surveillance Programme (IDSP); National Centre for Disease Control" Ministry of Health \& Family Welfare, Government of India; https://ncdc.gov.in/index1.php?lang=1\&level= 1 \&sublinkid=143\&lid=54.

22. Comprehensive Assessment of National Surveillance Systems in Sri Lanka; Joint Assessment Report; 4-13 March 2003; World Health Organization; Regional Office for South-East Asia; New Delhi.

23. Disease surveillance go online; Epidemiology Unit, Ministry of Health, \#231, De Saram Place, Colombo 10; http://www.epid.gov.lk/web/index.php?option= com_content\&view=article\&id=174\&Itemid= 489\&lang=en.

24. Chandrasekar K, et al. Notifiable disease surveillance in Sri Lanka and the United Kingdom: a comparative study. Sri Lanka J Bio Med Inform. 2013;4(1):14-22. https://doi.org/10.4038/sljbmi.v4i1.5190.

$25 . \bullet \quad$ Fall IS, Rajatonirina S, Yahaya AA, et al. Integrated Disease Surveillance and Response (IDSR) strategy: current status, challenges and perspectives for the future in Africa. BMJ Global Health. 2019;4:e001427. https://doi.org/10.1136/bmjgh-2019-001427

Effective integrated surveillance system described from LMICs.

26. Promoting dengue vector surveillance and control; https://www.who.int/activities/promoting-denguevector-surveillance-and-control.

27. Mwabukusi M, Karimuribo ED, Rweyemamu MM, Beda E. Mobile technologies for disease surveillance in humans and animals. Onderstepoort J Vet Res. 2014;81(2) art. \#737, 5 pages. https://doi.org/10. 4102/ojvr.v81i2.737.

28. Birkhead GS, Klompas M, Shah NR. Uses of electronic health records for public health surveillance to advance public health. Ann Rev Public Health. 2015;36:345-9 file://C:/Users/admin/Documents/research/ review\%20article\%20on\%20surveillance/ EHR\%20for\%20surveillance\%20annurev-publhealth031914-122747.pdf.

29. Circular No 01-13/2015; Quality Indicators related to Hospital Acquired Infections, ministry of Health, Sri Lanka; http://www.health.gov.lk/CMS/cmsmoh1/ viewcircular.php?cno=01-13/2015\&med=english.

30. Collection Staphylococcus aureus: guidance, data and analysis; https://www.gov.uk/government/collections/ staphylococcus-aureus-guidance-data-and-analysis.

31. Nejad SB, Allegranzi B, Syed SB, Ellis B, Pittet D. Health-care-associated infection in Africa: a systematic review; Bulletin of the World Health Organization; https://www.who.int/bulletin/volumes/89/10/11088179/en/.

32. Mahomed S, Ozayr M, Willem Sturm A, Knight S, Moodley P. Challenges with Surveillance of Healthcare-Associated Infections in Intensive Care Units in South Africa. Hindawi Crit Care Res Pract. 2017:7296317, 7 pages. https://doi.org/10.1155/ $2017 / 7296317$.

33. Types of Healthcare-associated Infections; Centers for Disease Control and Prevention (CDC); https://www. cdc.gov/hai/infectiontypes.html.

34.• European Centre for Disease Prevention and Control. European Surveillance of Clostridioides (Clostridium) difficile infections. Surveillance protocol version 2.4 . Stockholm: ECDC; 2019. https://www.ecdc.europa.eu/ en/publications-data/european-surveillance-

clostridium-difficile-infectionssurveillance-protocol-2. This describes how technically demanding surveillance is and the resources needed for an effective surveillance of certain infections.

35.• McDonald LC, Gerding DN, Johnson S, et al. Clinical Practice Guidelines for Clostridium difficile Infection in Adults and Children: 2017 Update by the Infectious Diseases Society of America (IDSA) and Society for Healthcare Epidemiology of America (SHEA). Clin Infect Dis. 2018;66(7):e1-e4.

This guideline illustrates the importance of proper diagnostic methods which is technically demanding.

36. Global Action Plan on Antimicrobial Resistance. World Health Organization. ISBN 978924150976 3; https:// 
www.who.int/antimicrobial-resistance/publications/ global-action-plan/en/.

37.• Jacobs J, Hardy L, Semret M, Lunguya O, Phe T, Affolabi D, et al. Diagnostic Bacteriology in District Hospitals in Sub-Saharan Africa: At the Forefront of the Containment of Antimicrobial Resistance. Front Med. 2019;6:205. https://doi.org/10.3389/fmed.2019. 00205

This illustrates the limitations in laboratory capacity in LMICs. 38. Ombelet S, Barbé B, Affolabi D, Ronat J-B, Lompo P, Lunguya $\mathrm{O}$, et al. Best practices of blood cultures in low and middle-income countries. Front Med. 2019;6:131. https://doi.org/10.3389/fmed.2019.00131.

39. Schubert S, Kostrzewa M. MALDI-TOF MS in the Microbiology Laboratory: Current Trends. Curr. Issues Mol. Biol. 2017;23:17-20. https://doi.org/10.21775/ cimb.023.017.

40. CLSI, editor. Performance Standards for antimicrobial susceptibility testing. $30^{\text {th }}$ ed. CLSI supplement L100. Wayne: Clinical and Laboratory Standards Institute; 2020.
41. Walters M, Lonsway D, Rasheed K, Albrecht V, McAllister S, Limbago B, et al. Investigation and control of Vancomycin-resistant Staphylococcus aureus: a guide for health departments and infection control personnel. Atlanta; 2015. Available at: http://www.cdc.gov/ hai/pdfs/VRSA-Investigation-Guide-05_12_2015.pdf

42. Joint External Evaluation of IHR Core Capacities Democratic Socialist Republic of Sri Lanka; Mission report: June 19-23, 2017; https://apps.who.int/iris/ bitstream/handle/10665/259266/WHO-WHE-CPIREP-2017.33-eng.pdf? sequence $=1$.

43. Global tuberculosis report 2019; ISBN 978-92-4156571-4; World Health Organization 2019.

\section{Publisher's Note}

Springer Nature remains neutral with regard to jurisdictional claims in published maps and institutional affiliations. 